BMJ Open

Diabetes

Research

\& Care

\section{Postprandial lipemia and the risk of coronary heart disease and stroke: the Atherosclerosis Risk in Communities (ARIC) Study}

To cite: Kats $D$, Sharrett $A R$, Ginsberg HN, et al.

Postprandial lipemia and the risk of coronary heart disease and stroke:

the Atherosclerosis Risk in Communities (ARIC) Study. BMJ Open Diabetes Research and Care 2017:5:e000335. doi:10.1136/bmjdrc-2016000335

Received 29 September 2016 Revised 1 December 2016 Accepted 11 December 2016

CrossMark

For numbered affiliations see end of article.

Correspondence to Dr Dmitry Kats;

dkats@email.unc.edu

\section{ABSTRACT}

Objective: Excessive levels of triglyceride-rich lipoproteins during postprandial lipemia (PPL) have been reported to be atherogenic. However, it is unclear whether the degree of PPL independently predicts cardiovascular disease (CVD) given the scarcity of longitudinal data with standardised measures of postprandial change. We reexamined associations of PPL with incident CVD events in a population-based cohort using detailed measures of postprandial change from a standardised fat challenge.

Research design and methods: Postprandial triglycerides, TG-rich lipoprotein triglycerides, retinyl palmitate and apolipoprotein B48 to B100 ratio were measured before (following a 12-hour fasting period) and after a fat-tolerance test meal in a middle-aged, biracial subcohort without CVD (coronary heart disease (CHD) or stroke) from the community-based Atherosclerosis Risk in Communities (ARIC) Study in 1990-1993. Using these measures, we estimated associations of postprandial change with incident CVD (CHD, stroke) through 2012. Stratified analyses by race, obesity and carotid atherosclerotic severity were also conducted.

Results: Of 559 participants, 127 (23\%) developed $\mathrm{CHD}$ and 27 (5\%) experienced a stroke over more than 20 years of follow-up. None of the measures of postprandial change were associated with incident CVD events in the overall sample, or by subgroups of race, obesity or carotid atherosclerotic severity (all $p>0.3$ ). Conclusions: The degree of PPL was not shown to predict excess CVD risk in extended follow-up of a population-based sample. While our study is the largest to examine the association between PPL and incident CVD using standardised postfat challenge measures, prospective investigation with similar assessment of PPL in more powered samples is warranted.

\section{INTRODUCTION}

Postprandial lipemia (PPL) refers to the metabolic state in which there is an increase of triglyceride-rich lipoproteins in circulation following fatty food consumption. While

\section{Significance of this study}

What is already known about this subject? The longitudinal association of postprandial lipemia with cardiovascular disease risk is not clear, stemming from limitations in how postprandial variables have been assessed in prospective studies.

What are the new findings?

There were no statistically significant associations between any of the multiple measures of postprandial change used in this study and incident cardiovascular outcomes.

How might these results change the focus of research or clinical practice?

More powered prospective studies using detailed, standardised measures of postprandial change are needed to elucidate the association of postprandial lipemia with the development of cardiovascular disease.

exaggerated levels of such triglycerides during PPL have been linked to prevalent atherosclerosis and cardiovascular disease (CVD) in case-control studies, ${ }^{1}{ }^{2}$ understanding of the association between PPL and incident atherothrombotic events is hampered by limitations in how PPL was assessed in previous longitudinal investigations. ${ }^{3-8}$ These studies, while extremely large in scale, used fasting or non-fasting postprandial triglycerides measured by a non-standardised protocol and at only one point in time.

Reliance on non-fasting triglycerides is flawed due to the high variability in their concentrations depending on the timing and contents of the last meal. Reported associations between fasting triglycerides and incident CVD events have been noticeably attenuated on adjustment for high-density lipoprotein cholesterol (HDL-C), ensuing debate in terms of whether excessive PPL confers any residual risk on the development of CVD beyond its relationship with HDL-C. ${ }^{7}$ 
Furthermore, concentrations of triglycerides taken at a single time point are not necessarily accurate indicators of postprandial change. ${ }^{9}$ Valid assessment of postprandial change is achieved instead through the synthesis of repeat measurements of postprandial moieties obtained over the course of a fat-tolerance test. ${ }^{9}$ Given the lack of prospective investigations with such detailed, standardised measures of postprandial change, the role PPL plays in CVD development is unclear.

Our study reassessed the longitudinal relationship between PPL and CVD using standardised fat challenge measures of postprandial change in a subsample from the population-based Atherosclerosis Risk in Communities (ARIC) Study. Associations of postprandial change with incident CVD events over $\sim 20$ years were estimated in white and African-American men and women. We further examined associations between these PPL measures and CVD risk by subgroups of race, body mass index (BMI) and baseline carotid intima media thickness (IMT), a measure of atherosclerosis severity.

\section{RESEARCH DESIGN AND METHODS}

\section{Study population}

The ARIC Study is an ongoing community-based cohort of 15792 men and women (mostly whites and African-Americans) aged 45-64 years at baseline (19871989) who were probabilistically selected from four US communities (Forsyth County, North Carolina; Jackson, Mississippi; suburbs of Minneapolis, Minnesota and Washington County). ${ }^{10}$ Our study sample originated from 1114 ARIC participants invited into a nested casecontrol study during ARIC visit 2, which occurred from 1990 to $1993 .{ }^{111}$ This substudy, composed of cases with subclinical carotid atherosclerosis and controls with minimal or no carotid artery atherosclerosis, was designed to examine the relationship between PPL and subclinical atherosclerosis, among participants without prevalent CVD. ${ }^{111}$

\section{Fat-tolerance test}

After a 12-hour period of fasting and avoidance of exercise, fasting blood specimens were collected. A liquid test meal was then administered, which consisted of heavy whipping cream, ice cream, safflower oil, chocolate syrup and powdered protein (Promod, Ross Laboratories) and contained $1265 \mathrm{kcal}, 32 \mathrm{~g}$ protein, $48 \mathrm{~g}$ carbohydrate, $105 \mathrm{~g}$ fat (52 g saturated) and $300 \mathrm{mg}$ cholesterol, in addition to $100000 \mathrm{IU}$ vitamin A (Aquasol, Armour Pharmaceutical Co.) for each $2 \mathrm{~m}^{2}$ of body surface area. As a safeguard against potential lactose intolerance, Lactaid was added to the meal. Participants were allotted $15 \mathrm{~min}$ to consume the test meal and instructed to ingest only water, unsweetened coffee or tea or sugarless soft drinks for 8 hours thereafter. Blood specimens were drawn 3.5 and 8 hours following test meal consumption. The number of postprandial blood collections (ie, the difference between two and four measurements) has shown minimal influence on the classification of postprandial response. ${ }^{2}$ Hence, measurements of PPL variables taken post-fasting and then 3.5 and 8 hours after consumption of the test meal should be suitable to assess postprandial response to a fatty meal over an 8-hour time period.

Blood specimens were centrifuged for $20 \mathrm{~min}(1500 \mathrm{~g}$, at $4^{\circ} \mathrm{C}$ ) to separate plasma. Plasma samples were shipped on dry ice and stored in liquid nitrogen at the central laboratory until analysis within a few days. Details of this procedure have been described elsewhere. ${ }^{111}$

\section{PPL assays}

Variation in CVD risk by subclasses of PPL-related variables was considered by using four separate measures of postprandial markers (each assessed at visit 2): postprandial triglycerides (TG), TG-rich lipoprotein triglycerides (TRL-TG), retinyl palmitate (RP) and apolipoprotein B48 to apolipoprotein $\mathrm{B} 100$ ratio (apoBR). TG and TRL-TG appear in the 'top fraction' and are markers of very low-density lipoproteins, whereas measures of RP and apoBR are surrogates for chylomicrons. In this study, TG levels were measured enzymatically, TRL-TG samples were isolated by ultracentrifugation, ${ }^{12}$ plasma RP levels were determined using high-pressure liquid chromatography ${ }^{13}$ and apoBR values were ascertained by sodium dodecyl sulfate electrophoresis. ${ }^{14}$

To yield a measure of postprandial change, the incremental area under the curve (iAUC) defined by each PPL parameter across the available time points $(0,3.5$ and 8 hours after the test meal) was calculated, with negative results set to $0 .{ }^{1}$ For the purposes of this study, the iAUC can be interpreted as the two-dimensional postprandial response over 8 hours. The iAUC measures were transformed into weighted SD units to reflect the sampling weights of the overall ARIC sample from which case-control participants were drawn. Weighted SD units can be interpreted in the same manner as conventional SD units.

\section{Other covariates}

HDL-C and low-density lipoprotein cholesterol (LDL-C) were measured at visit $2 .{ }^{15} 16$ Trained ARIC personnel used a standardised protocol to measure height, weight, former as well as current cigarette smoking status and diastolic and systolic blood pressure at visit $2 .{ }^{17}$ Prevalent hypertension was indicated by a systolic blood pressure $\geq 140 \mathrm{~mm} \mathrm{Hg}$, a diastolic blood pressure $\geq 90 \mathrm{~mm} \mathrm{Hg}$ and/or self-reported use of antihypertensive medication. The use of lipid-lowering medications was self-reported by the patient and verified by ARIC personnel (checking of medications brought by the patient) at visit 2. Fasting blood glucose measures were also taken at visit 2 .

Severity of atherosclerosis was assessed using B-mode ultrasound as in the aforementioned nested case-control study during visit 2. ${ }^{111}$ In that study, white participants with IMT values above the 95th centile of 
the ARIC distribution were sampled as cases, while the 90th centile was applied to obtain a sufficient number of cases among black participants. Controls in race/ethnicity groups were below the 75th centile of IMT in all artery segments evaluated, as IMT values greater than the 75th centile are considered abnormal and indicative of increased CVD risk. ${ }^{18}$ Cases from that study were classified as having a high severity of atherosclerosis in this study, and controls as having a low severity of atherosclerosis.

\section{Ascertainment of CVD}

Incident CHD events included definite or probable myocardial infarction (MI), 'silent' MI per centrally interpreted ECG, definite CHD death or coronary revascularisation. This information was obtained through annual contact of participants, yearly discharge lists from local hospitals and ongoing survey of death certificates. ${ }^{17}$ Incident (ischaemic) stroke was identified through hospital discharge codes and deaths attributed to stroke. ${ }^{19}$ Physician reviewers adjudicated all possible CHD and stroke events and classified them as definite, probable or non-events based on information abstracted from medical records. ${ }^{19}$ Cohort follow-up of incident CHD and stroke events through 31 December 2012 was included in these analyses.

\section{Statistical analyses}

Plots of residuals depicted a relatively linear relationship between each PPL parameter (as the iAUC-transformed weighted SD measure) and incident CVD (CHD or stroke). For each PPL parameter, we therefore estimated crude, age-sex adjusted and fully adjusted associations between an increase of 1-unit in the iAUC-transformed weighted SD measure and incident CVD events (CHD individually, stroke individually, and CHD or stroke as a merged outcome). Cox proportional hazard models ${ }^{20}$ were used to quantify estimates of associations on the relative scale via HRs, and incident rate differences (IRDs) from Poisson models ${ }^{21}$ were used as estimates on the absolute scale.

Since knowledge of the biological mechanism involved in postprandial change and CVD development is not clear, we applied stepwise selection $(\mathrm{p}<0.15$ for inclusion and exclusion) - as opposed to a priori adjustment- to guide in the choice of covariates for fully adjusted models. The following variables, each at visit 2, were considered in such models: age, sex, race (white or African-American), study center location, BMI ( $\geq 30 \mathrm{~kg} /$ $\mathrm{m}^{2}$ or $<30 \mathrm{~kg} / \mathrm{m}^{2}$ ), atherosclerosis severity (high or low), HDL-C, LDL-C, fasting blood glucose, current cigarette smoking, former cigarette smoking, prevalent hypertension and use of lipid-lowering medications.

As per findings from the ARIC case-control study of PPL and atherosclerosis, ${ }^{1}$ we also estimated associations of our PPL measures with incident CVD by dichotomies of race, BMI and atherosclerosis severity. Comparison of effect measures across these subgroups of low versus high cardiometabolic risk may provide insights into the temporality and etiology of potential mechanisms. For instance, evidence of an independent association between excessive PPL and incident CVD in participants with low but not high IMT would suggest that PPL exerts its role in the pathophysiology of CVD prior to or during the earlier stages of atherosclerosis. In this study, all statistical analyses were performed using SAS V.9.3 (SAS Corp, Cary, North Carolina, USA).

\section{RESULTS}

Of 1114 participants invited into the original ARIC case-control study, 555 individuals were excluded from this study (84 declined participation, 195 had prevalent CVD, 179 had other medical exclusions and 97 had test meal intolerance). Our final analytic sample consisted of 559 participants-228 (41\%) women and 148 (26\%) African-Americans distributed similarly across the four study sites. Baseline characteristics of participants are presented in table 1 .

The mean values of the iAUC-transformed weighted SD measures of PPL at visit 2 were 1.7 for TG, 1.4 for TRL-TG, 1.9 for RP and 1.4 for apoBR. The means of the iAUC PPL measures prior to weighted SD transformation were $708 \mathrm{mg} / \mathrm{dL} \times$ hour $(\mathrm{SD}=473)$ for $\mathrm{TG}, 536 \mathrm{mg} /$ $\mathrm{dL} \times$ hour $\quad(\mathrm{SD}=432)$ for $\quad \mathrm{TRL}-\mathrm{TG}, \quad 5781 \mu \mathrm{g} / \mathrm{L} \times \mathrm{hr}$ $(\mathrm{SD}=3051)$ for $\mathrm{RP}$ and 15 hour $(\mathrm{SD}=12)$ for apoBR. Before iAUC calculations were performed, the mean values of the PPL parameters were $121 \mathrm{mg} / \mathrm{dL}(\mathrm{SD}=60)$ for $\mathrm{TG}, \quad 71 \mathrm{mg} / \mathrm{dL} \quad(\mathrm{SD}=54)$ for TRL-TG, $19 \mu \mathrm{g} / \mathrm{L}$ $(\mathrm{SD}=36)$ for $\mathrm{RP}$ and $3.0(\mathrm{SD}=1.8)$ for apoBR.

A total of 127 (23\%) incident cases of CHD and 27 (5\%) incident cases of stroke accrued in the study sample by the end of 2012 (median follow-up=20.5 years). The study was sufficiently powered (at $80 \%$ ) to detect an $\mathrm{HR}=1.3$ in the overall sample. However, analyses in the overall sample did not reveal any significant associations between the PPL measures and incident CHD or stroke (table 2). Point estimates of effect sizes (HRs and IRDs) were generally null, even in crude models. As interpreted using the 95\% CIs of fully adjusted HRs, estimates of the excess CVD risk (attributed to each 1-unit increase in the iAUC-transformed weighted SD of RP, in this case) are not expected to surpass $28 \%$ in $95 \%$ of study repetitions.

In stratified analyses, the smallest effect size capable of being detected with sufficient power was an $\mathrm{HR}=1.6$ (in the subgroup of white participants). Similar to as in the overall sample, no significant associations between the PPL measures and incident CVD events were observed by race, BMI category or atherosclerosis severity (all $p>0.4$ ). Point estimates of the associations between our PPL measures and CVD from each of these subgroup models were centered around the null value in a similar fashion as in the overall sample. Given the relatively smaller sample size of each subgroup compared to the overall sample, as expected, estimates were also less 
Table 1 Characteristics of study participants $(\mathrm{N}=559)$ from the ARIC nested case-control study of PPL and atherosclerosis during 1990-1993 by tertiles of the AUC-transformed weighted SD measure of postprandial triglycerides (TG)

\begin{tabular}{|c|c|c|c|}
\hline \multirow[b]{2}{*}{ Covariates } & \multicolumn{3}{|l|}{ TG tertile } \\
\hline & 1 & 2 & 3 \\
\hline Age mean (SD), years & $57.9(5.6)$ & $58.6(5.3)$ & $58.1(5.4)$ \\
\hline \multicolumn{4}{|l|}{ Sex } \\
\hline Female, n (\%) & $103(56)$ & $69(37)$ & $54(29)$ \\
\hline Male, n (\%) & $82(44)$ & $116(63)$ & $131(71)$ \\
\hline \multicolumn{4}{|l|}{ Race } \\
\hline African-American, n (\%) & $47(25)$ & $48(26)$ & $50(27)$ \\
\hline White, n (\%) & $138(75)$ & $137(74)$ & $135(73)$ \\
\hline \multicolumn{4}{|l|}{ Study centre } \\
\hline Forsyth, NC, n (\%) & $34(18)$ & $47(25)$ & $59(32)$ \\
\hline Jackson, MS, n (\%) & $46(25)$ & $46(25)$ & $48(26)$ \\
\hline Minneapolis, MN, n (\%) & $63(34)$ & $43(23)$ & $44(24)$ \\
\hline Washington County, MD, n (\%) & $42(23)$ & $49(27)$ & $34(18)$ \\
\hline \multicolumn{4}{|l|}{ BMI } \\
\hline$\geq 30 \mathrm{~kg} / \mathrm{m}^{2}, \mathrm{n}(\%)$ & $29(16)$ & $42(23)$ & $42(23)$ \\
\hline$<30$ kg/m², n (\%) & $156(84)$ & $143(77)$ & $143(77)$ \\
\hline \multicolumn{4}{|l|}{ Carotid IMT level } \\
\hline High, n (\%) & $52(28)$ & $70(38)$ & $80(43)$ \\
\hline Low, n (\%) & $133(72)$ & $115(62)$ & $105(57)$ \\
\hline HDL-C mean (SD), mg/dL & $14.2(8.5)$ & $10.3(6.4)$ & $8.2(4.8)$ \\
\hline LDL-C mean (SD), mg/dL & $124(32)$ & $131(36)$ & $139(31)$ \\
\hline Fasting blood glucose mean (SD), mg/dL & $108(36)$ & $111(38)$ & $108(30)$ \\
\hline \multicolumn{4}{|l|}{ Current cigarette smoking } \\
\hline Smoker, n (\%) & $40(22)$ & $48(26)$ & $50(27)$ \\
\hline Non-smoker, n (\%) & $145(78)$ & $136(74)$ & $135(73)$ \\
\hline \multicolumn{4}{|l|}{ Former cigarette smoking } \\
\hline Smoker, n (\%) & $67(36)$ & $71(39)$ & $81(44)$ \\
\hline Non-smoker, n (\%) & $118(64)$ & $113(61)$ & $104(56)$ \\
\hline \multicolumn{4}{|l|}{ Prevalent hypertension } \\
\hline Present, n (\%) & $52(28)$ & $48(26)$ & $49(27)$ \\
\hline Absent, n (\%) & $133(72)$ & $136(74)$ & $135(73)$ \\
\hline
\end{tabular}

precise in stratified analyses. For brevity, quantitative results from stratified analyses are not depicted.

\section{CONCLUSIONS}

Extended follow-up of a well-characterised biracial sample of men and women did not reveal evidence of a relationship between exaggerated PPL response and increased incidence of CVD events. Further, no associations between the extent of PPL and incident CVD were observed by obesity status or level of carotid atherosclerosis.

Given the lack of statistically significant associations between our comprehensive measures of PPL and CVD risk, together with the consistently null-sized estimates of these associations, the results of this study suggest that PPL does not influence the risk of atherothrombotic events in a discernible way. Our negative findings mimic those from case-control studies that used a similar lipid load and postchallenge PPL measures, ${ }^{2}{ }^{22}$ but they are not consistent with the significant (albeit modest) associations reported in considerably more powered prospective investigations. ${ }^{3-8}$ However, these large-scale prospective studies were limited to non-standardised measures of fasting or non-fasting triglycerides assessed at only one time point. Our investigation was able to assess lipemic response over time using diverse lipoprotein and apolipoprotein measurements from a standardised fat challenge.

Equally important to acknowledge is the low statistical power of this study. Notwithstanding this limitation, until now, this is the largest and most extensive populationbased study to examine the longitudinal association between PPL and CVD using standardised postchallenge lipid moieties as measures of PPL. Our findings ultimately point to excessive PPL not being as strong an independent predictor of CVD risk as previously indicated. Nevertheless, on the population level, PPL may be an important overall risk determinant and therapeutic target which influences other CVD risk factors such as HDL-C. Better powered prospective studies with detailed, standardised measures of postprandial change are needed to clarify the role (if any) of excessive PPL in CVD pathophysiology. 
Table 2 HR and IRD estimates (with 95\% Cls and p values) for associations between an increase of 1-unit in the iAUC-transformed weighted SD measure of each PPL parameter and incident CHD or stroke

\begin{tabular}{|c|c|c|c|c|c|c|c|}
\hline PPL parameter & Model & $\mathbf{N}$ & Events & HR $(95 \% \mathrm{Cl})$ & p Value & IRD* (95\% Cl) & p Value \\
\hline \multirow[t]{3}{*}{ TG } & Crude & 534 & 123 & 0.94 (0.80 to 1.11$)$ & 0.459 & $-4.1(-12.4$ to 5.0$)$ & 0.382 \\
\hline & Age and sex & 534 & 123 & 0.93 (0.80 to 1.10$)$ & 0.414 & $-3.3(-14.2$ to 3.9$)$ & 0.386 \\
\hline & Fully adjusted $†$ & 519 & 117 & 0.95 (0.80 to 1.13$)$ & 0.557 & $-3.8(-15.3$ to 4.2$)$ & 0.541 \\
\hline \multirow[t]{3}{*}{ TRL-TG } & Crude & 495 & 112 & 0.97 (0.82 to 1.15$)$ & 0.710 & $-2.2(-10.1$ to 6.5$)$ & 0.617 \\
\hline & Age and sex & 495 & 112 & 0.96 (0.81 to 1.15$)$ & 0.662 & $-2.0(-11.3$ to 6.4$)$ & 0.588 \\
\hline & Fully adjusted $†$ & 480 & 106 & 0.98 (0.81 to 1.18$)$ & 0.828 & $-1.4(-11.6$ to 7.1$)$ & 0.674 \\
\hline \multirow[t]{3}{*}{ RP } & Crude & 534 & 122 & 1.06 (0.89 to 1.25$)$ & 0.532 & $2.3(-5.7$ to 10.6$)$ & 0.558 \\
\hline & Age and sex & 534 & 122 & 1.05 (0.89 to 1.25$)$ & 0.565 & $1.1(-4.5$ to 9.2$)$ & 0.552 \\
\hline & Fully adjusted $†$ & 519 & 116 & 1.07 (0.90 to 1.28$)$ & 0.437 & $1.3(-5.2$ to 9.4$)$ & 0.517 \\
\hline \multirow[t]{3}{*}{ apoBR } & Crude & 485 & 112 & 0.91 (0.76 to 1.10$)$ & 0.340 & $-1.2(-9.8$ to 7.5$)$ & 0.679 \\
\hline & Age and sex & 485 & 112 & 0.91 (0.76 to 1.10$)$ & 0.344 & $-1.3(-11.0$ to 7.3$)$ & 0.698 \\
\hline & Fully adjusted $\ddagger$ & 470 & 106 & 0.93 (0.77 to 1.13$)$ & 0.460 & $-1.5(-12.3$ to 6.7$)$ & 0.706 \\
\hline
\end{tabular}

${ }^{*}$ Estimate of IRD per 1000 person-years.

†Adjusted for age, sex, race and fasting blood glucose, following stepwise selection.

$\ddagger$ Adjusted for age, sex, race, fasting blood glucose and current smoking status, following stepwise selection.

apoBR, apolipoprotein B100 ratio; IRD, incident rate difference; PPL, postprandial lipemia; RP, retinyl palmitate; TG, postprandial triglycerides;

TRL-TG, TG-rich lipoprotein triglycerides.

\section{Author affiliations}

'Department of Epidemiology, Gillings School of Global Public Health, University of North Carolina at Chapel Hill, Chapel Hill, North Carolina, USA ${ }^{2}$ Department of Epidemiology, Johns Hopkins Bloomberg School of Public Health, Baltimore, Maryland, USA

${ }^{3}$ Irving Institute for Clinical and Translational Research, Columbia College of Physicians and Surgeons, New York, New York, USA

${ }^{4}$ Michael E Debakey Veterans Affairs Hospital, Houston, Texas, USA

${ }^{5}$ Section of Cardiovascular Research, Department of Medicine, Baylor College of Medicine, Houston, Texas, USA

${ }^{6}$ Houston Methodist Debakey Heart and Vascular Center, Houston, Texas, USA

Acknowledgements The authors thank the staff and participants of the ARIC Study for their important contributions.

Contributors DK performed the statistical evaluation of the data and wrote the manuscript. ARS designed the original study protocol, directed the study and provided input throughout the manuscript preparation. HNG, VN, CMB and $\mathrm{RCH}$ provided expert input and critical review throughout the manuscript preparation. GH coordinated the study, provided input during manuscript preparation and provided funding. All authors approved the final manuscript.

Funding This work was supported by National Heart, Lung, and Blood Institute contracts (HHSN268201100005C, HHSN268201100006C, HHSN268201100007C, HHSN268201100008C, HHSN268201100009C, HHSN268201100010C, HHSN268201100011C and HHSN268201100012C)

Disclaimer The views expressed in this article are those of the authors and do not necessarily represent the views of the Department of Veterans Affairs.

Competing interests HNG reports grants from National Institutes of Health USA, during the conduct of the study. VN reports personal fees from Siemens Laboratory, other from Roche, outside the submitted work; and Coinvestigator on a provisional patent filed along with Baylor College of Medicine and Roche for 'Biomarkers to improve prediction of heart failure.' CMB reports personal fees from AstraZeneca, grants and personal fees from Amarin, grants and personal fees from Amgen, grants and personal fees from Eli Lilly, grants and personal fees from Esperion, personal fees from Matinas BioPharma, personal fees from Merck, grants and personal fees from Novartis, grants and personal fees from Pfizer, grants and personal fees from Regeneron, grants and personal fees from Sanofi-Synthelabo, grants from National Institutes of Health, grants from American Heart Association, grants from American Diabetes Association, grants and personal fees from Ionis, outside the submitted work. RCH reports grants from Denka Seiken Co, outside the submitted work; In addition, RCH has a patent 'Biomarkers to Improve Prediction of Heart Failure Risk' (patent no. 61721475) pending.
Ethics approval Institutional Review Boards from all participating institutions approved the study.

Provenance and peer review Not commissioned; externally peer reviewed.

Data sharing statement No additional data are available.

Open Access This is an Open Access article distributed in accordance with the Creative Commons Attribution Non Commercial (CC BY-NC 4.0) license, which permits others to distribute, remix, adapt, build upon this work noncommercially, and license their derivative works on different terms, provided the original work is properly cited and the use is non-commercial. See: http:// creativecommons.org/licenses/by-nc/4.0/

\section{REFERENCES}

1. Sharrett AR, Chambless LE, Heiss G, et al. ARIC investigators. Association of postprandial triglyceride and retinyl palmitate responses with asymptomatic carotid artery atherosclerosis in middle-aged men and women the Atherosclerosis Risk in Communities (ARIC) study. Arterioscler Thromb Vasc Biol 1995;15:2122-9.

2. Ginsberg HN, Jones J, Blaner WS, et al. Association of postprandial triglyceride and retinyl palmitate responses with newly diagnosed exercise-induced myocardial ischemia in middle-aged men and women. Arterioscler Thromb Vasc Biol 1995;15:1829-38.

3. Angelantonio E, Sarwar N, Perry P, et al. Major lipids, apolipoproteins, and risk of vascular disease. JAMA 2009:302:1993-2000.

4. Bansal S, Buring JE, Rifai N, et al. Fasting compared with nonfasting triglycerides and risk of cardiovascular events in women. JAMA 2007:298:309-16.

5. Lindman AS, Veierød MB, Tverdal A, et al. Nonfasting triglycerides and risk of cardiovascular death in men and women from the Norwegian Counties Study. Eur J Epidemiol 2010;25:789-98.

6. Nordestgaard BG, Benn M, Schnohr P, et al. Nonfasting triglycerides and risk of myocardial infarction, ischemic heart disease, and death in men and women. JAMA 2007;298:299-308.

7. Sarwar N, Danesh J, Eiriksdottir G, et al. Triglycerides and the risk of coronary heart disease 10158 incident cases among 262525 participants in 29 western prospective studies. Circulation 2007;115:450-8.

8. Hokanson JE, Austin MA. Plasma triglyceride level is a risk factor for cardiovascular disease independent of high-density lipoprotein cholesterol level: a metaanalysis of population-based prospective studies. J Cardiovasc Risk 1996;3:213-19.

9. Maraki M, Aggelopoulou N, Christodoulou N, et al. Validity of abbreviated oral fat tolerance tests for assessing postprandial lipemia. Clinical Nutrition 2011;30:852-7. 
10. The ARIC Investigators. The Atherosclerosis Risk in Communities (ARIC) Study: design and objectives. Am J Epidemiol 1989;129:687-702.

11. Sharrett AR, Heiss G, Chambless LE, et al. Metabolic and lifestyle determinants of postprandial lipemia differ from those of fasting triglycerides the Atherosclerosis Risk in Communities (ARIC) study. Arterioscler Thromb Vasc Biol 2001;21:275-81.

12. Hainline A Jr, Karon J, Lippel K, eds. Manual of laboratory operations: lipid and lipoprotein analysis. Bethesda (MD): National Heart, Lung and Blood Institute, Lipid Research Clinics Program, 1982 [HEW Pub. No. (NIH) 75-628 (rev), US Government Printing Office Publication No. 1982-361-132:678.].

13. Boerwinkle E, Brown S, Sharrett AR, et al. Apolipoprotein E polymorphism influences postprandial retinyl palmitate but not triglyceride concentrations. Am J Hum Genet 1994;54:341.

14. Schonfeld G, Lees RS, George PK, et al. Assay of total plasma apolipoprotein B concentration in human subjects. J Clin Invest 1974;53:1458-67.

15. Warnick GR, Benderson J, Albers JJ. Dextran sulfate- $\mathrm{Mg}^{2+}$ precipitation procedure for quantitation of high density-lipoprotein cholesterol. Clin Chem 1983:10:91-9.

16. Friedewald WT, Levy RI, Fredrickson DS. Estimation of the concentration of low-density lipoprotein cholesterol in plasma, without use of the preparative ultracentrifuge. Clin Chem 1972;18:499-502.

17. White AD, Folsom AR, Chambless LE, et al. Community surveillance of coronary heart disease in the Atherosclerosis Risk in Communities (ARIC) Study: methods and initial two years' experience. J Clin Epidemiol 1996;49:223-33.

18. Stein $\mathrm{JH}$, Korcarz CE, Hurst RT, et al. Use of carotid ultrasound to identify subclinical vascular disease and evaluate cardiovascular disease risk: a consensus statement from the American Society of Echocardiography Carotid Intima-Media Thickness Task Force endorsed by the Society for Vascular Medicine. J Am Soc Echocardipgr 2008;29:93-111.

19. Jones SA, Gottesman RF, Shahar E, et al. Validity of hospital discharge diagnosis codes for stroke the atherosclerosis risk in communities study. Stroke 2014;45:3219-25.

20. Therneau TM, Grambsch PM. Modeling survival data: extending the Cox model. Springer Science \& Business Media, 2000.

21. Littell RC, Stroup WW, Milliken GA, et al. SAS for mixed models. SAS Institute, 2006.

22. Reyes-Soffer G, Holleran S, Karmally W, et al. Measures of postprandial lipoproteins are not associated with coronary artery disease in patients with type 2 diabetes mellitus. J Lipid Res 2009;50:1901-9. 